\title{
Manipulation of single atoms and molecules by electron probe and mechanical force
}

\author{
Nan Yao, Guangming Cheng and Pengcheng Chen
}

Princeton Institute for the Science and Technology of Materials, Princeton University, Princeton, New Jersey, United States

Nan Yao, Guangming Cheng and Pengcheng Chen

Princeton Institute for the Science and Technology of Materials, Princeton University, Princeton, NJ 08544, USA

Email: nyao@princeton.edu, gcheng2@princeton.edu and pengchengchen@ princeton.edu

Science and technology ever seek to build structures of progressively smaller size that has finally reached the point where structures and materials can be built via atom-by-atom engineering. The ability of manipulation of single atoms or molecules can enable us to build and characterize model systems that will advance the material development and the understanding of physics and chemistry.[1-4] By necessity, we must combine both the imaging and manipulation techniques, to affect matter and to real-time monitor the system at atomic level. The scanning transmission electron microscopy (STEM), based on the atomically focused electron probe [2], can be utilized to introduce subtle changes in atomic structure while the process is resolved at atomic level, including vacancy ordering, single dopant atom motion and chemical reactions. On the other hand, the atomic force microscopy (AFM) which has made it possible to image the surfaces of materials with picometre $(\mathrm{pm})$ resolution, and also to manipulate single atoms and individual chemical bonds [1]. Besides bond formation, the controlled rupture of a chemical bond using mechanical forces, along with accurate measurements of these forces, provides new insights into the physical nature of a chemical bond. Here, we present a few examples of imaging or manipulating single atoms and molecules, which include (1) imaging the continuous movement of Au single atoms on a graphene substrate under electron beam, and (2) manipulating a single carbon monoxide $(\mathrm{CO})$ molecule on a ferrous phthalocyanine (FePc) complex via mechanical forces applied by AFM tips.

Figure 1 shows a series of snapshots from the recording of Au singe atoms' movement under electron beam. Each frame shown in the figure was taken $0.6 \mathrm{~ms}$ interval. The Au single atoms were randomly dispersed on a graphene substrate. Electron beam provided the kinetic energy to drive the single atoms moving (or vibrating) around. We are keeping interests in exploring this phenomenon as the change of experimental conditions. The tracked trajectories give direct visualization of the single gold atoms movements. And the number of tracked single atoms in each frame is quantified.

Figure 2 shows the breaking of a single dative bond between a $\mathrm{CO}$ molecule and a ferrous phthalocyanine $(\mathrm{FePc})$ complex by applying mechanical forces using the scanning tip of AFM. The dative $\mathrm{CO}-\mathrm{FePc}$ bond is known to be formed via s-donation from the $\mathrm{CO} 5 \mathrm{~s}$ orbital and p-back donation from $\mathrm{Fe}$ dp. By decreasing the tip height, the repulsive interactions increased, as indicated by the increased contrast in the images. Upon further reducing the tip height $(+30 \mathrm{pm})$, a sudden change of the image occurred during scanning, as indicated by a line created with a different contrast. Subsequent scans showed the repulsion had disappeared, indicating that the $\mathrm{CO}$ attached to FePc was dislodged due to the strong repulsion with the tip. Comparison of the contrast in the lower part to the upper part of the same AFM image (iv) obtained at the same tip height reveals a downward shift of FePc by $\sim 30 \mathrm{pm}$ upon CO removal. 
This observation confirmed the rupture of the dative bond between $\mathrm{CO}$ and FePc induced by the increased interactions during tip scanning and the bonding nature between FePc and the substrate.[5]
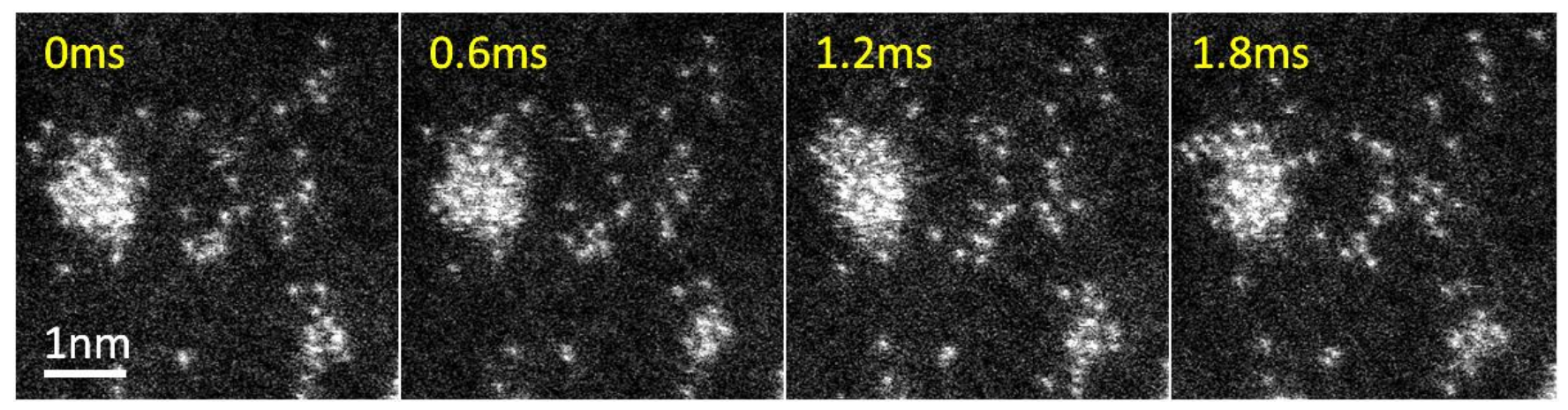

Figure 1. Imaging of $\mathrm{Au}$ single atoms under scanning transmission electron microscope. Au atoms keep moving under electron beam.

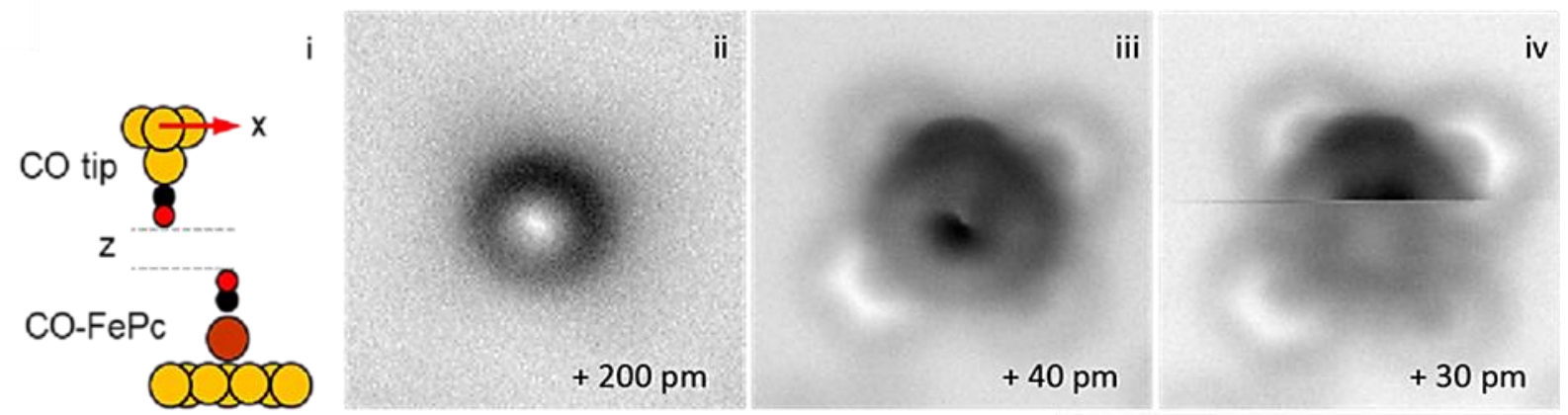

Figure 2. Rupturing the dative CO-FePc bond using AFM tips. (i) Schematic of a CO-AFM tip interacting with $\mathrm{CO}-\mathrm{FePc}(\mathrm{Cu}$ : yellow; carbon: black; oxygen: red; Fe: orange) and (ii-iv) showing noncontact AFM images obtained at different tip heights $(\mathrm{z})$ and the final dislodging of $\mathrm{CO}$ at $\mathrm{z}=+30 \mathrm{pm}$.

\section{References}

[1] O. Custance, R. Perez, S. Morita, Atomic force microscopy as a tool for atom manipulation, Nature nanotechnology 4(12) (2009) 803-810.

[2] O. Dyck, S. Kim, S.V. Kalinin, S. Jesse, Placing single atoms in graphene with a scanning transmission electron microscope, Applied Physics Letters 111(11) (2017) 113104.

[3] P. Chen, J.N. Metz, A.S. Mennito, S. Merchant, S.E. Smith, M. Siskin, S.P. Rucker, D.C. Dankworth, J.D. Kushnerick, N. Yao, Petroleum pitch: Exploring a 50-year structure puzzle with real-space molecular imaging, Carbon 161 (2020) 456-465.

[4] P. Chen, S. Fatayer, B. Schuler, J.N. Metz, L. Gross, N. Yao, Y. Zhang, The Role of Methyl Groups in the Early Stage of Thermal Polymerization of Polycyclic Aromatic Hydrocarbons Revealed by Molecular Imaging, Energy \& Fuels 35(3) (2020) 2224-2233.

[5] The authors acknowledge the use of Princeton's Imaging and Analysis Center (IAC), which is partially supported by the Princeton Center for Complex Materials (PCCM), a National Science Foundation (NSF) Materials Research Science and Engineering Center (MRSEC; DMR-2011750). 